\title{
Adaptaciones Metodológicas en el Aula de Lengua Extranjera para el Alumnado con Discapacidad Intelectual Leve: Estudio de Caso
}

\author{
Leopoldo Medina SÁnchez \\ Cristina Pérez Valverde \\ Universidad de Granada
}

Recibido: 28 junio 2017 / Aceptado: 8 noviembre 2017

ISSN: $1697-7467$

\begin{abstract}
RESUMEN: El objetivo de este artículo es describir qué enfoques metodológicos, dinámicas de clase y material didáctico adaptado se utilizan para el aprendizaje del inglés de una alumna con discapacidad intelectual leve inscrita en un centro escolar de Educación Secundaria. Del mismo modo, analizamos la valoración de las condiciones de trabajo de la alumna realizada por sus docentes de inglés. Para ello, hemos llevado a cabo un estudio de caso. Las herramientas de recogida de datos empleadas son: análisis documental, observación participante y entrevistas en profundidad. El análisis de los resultados revela la primacía de una metodología de enseñanza tradicional en los enfoques metodológicos y dinámicas de clase observados y la necesidad de una mejora del material didáctico adaptado. Asimismo, la valoración de las docentes sobre las condiciones de trabajo pone de manifiesto, entre otras, la necesidad de una ratio de alumnado menos numerosa y de mayores recursos humanos e infraestructurales, los problemas de absentismo de la alumna y la importancia de una colaboración más estrecha entre el centro escolar y la familia.

Palabras clave: metodologías de enseñanza de inglés lengua extranjera (ILE), discapacidad intelectual leve, inclusión, necesidades educativas especiales.
\end{abstract}

Methodological Adaptations In The Foreign Language Classroom For Students With Mild Intellectual Disability: A Case Study

ABSTRACT: The purpose of this paper is to describe the methodological approaches, classroom dynamics, and teaching material employed for the EFL learning of a student with mild intellectual disability at a secondary school, as well as to elicit the appraisal and judgment of the student's working conditions on the part of her English teachers. To this end, we have conducted a case study based on documentary analysis, in-depth interviews, and participant observation. The results of the analysis manifest the adoption of an eclectic approach in which traditional methodologies play a major role, and the need to improve the teaching material specifically adapted for the student. Moreover, the discourse of the teachers reveals the urgency for a smaller class size, a greater amount of human and infrastructural resources, problems regarding the student's absenteeism, and the importance of promoting a close collaboration between the school and the student's family.

Keywords: ELT methodologies, mild intellectual disability, inclusion, special educational needs. 


\section{INTRODUCCIÓN}

Hoy en día, según nuestra legislación educativa vigente en materia de inclusión, la práctica escolar de los docentes de lengua extranjera (LE) en centros escolares ordinarios debe estar en consonancia con los criterios de calidad y equidad propios de una educación inclusiva (Ley Orgánica para la Mejora de la Calidad Educativa, LOMCE, 2013). Atender a la diversidad de necesidades del alumnado conlleva garantizar el derecho fundamental a una educación igualitaria, además del reconocimiento y el desarrollo de sus capacidades individuales con el fin de que formen parte de la sociedad del mañana en calidad de ciudadanos de pleno derecho (Declaración Universal de Derechos Humanos, 1948). Para dicho fin, las metodologías de enseñanza han de responder a la diversidad de estilos y ritmos de aprendizaje del alumnado.

Según recoge la Ley Orgánica de Educación de 2006 en los artículos 71-73 que no han sido modificados y, por tanto, se mantienen en la LOMCE, el alumnado con discapacidad intelectual leve se clasifica dentro de la categoría general: "Alumnado con Necesidades Educativas de Apoyo Específico" (ACNEAE), que es aquel que requiere una atención educativa diferente a la ordinaria por presentar: a) dificultades específicas de aprendizaje; b) altas capacidades intelectuales; c) incorporación tardía al Sistema Educativo español; d) condiciones personales; e) historia escolar; f) presentar Necesidades Educativas Especiales (NEE). En esta última categoría se incluye si han sido adecuadamente diagnosticados al alumnado con discapacidad intelectual leve aunque este pueda presentar simultáneamente características propias de las otras categorías anteriormente citadas. Consideramos necesaria la investigación en torno a la provisión de apoyos socioeducativos al alumnado con discapacidad intelectual leve en el aula de inglés como lengua extranjera, debido a la diversidad de perfiles de aprendizaje que a menudo en ella se presentan por un lado y, por otro lado, debido a la insuficiencia de recursos teóricos y prácticos de los que dispone el profesorado de inglés a la hora de conocer y poner en práctica metodologías y estrategias de enseñanza destinadas a ofrecer una respuesta educativa equitativa y de calidad a este alumnado.

Por esta razón, a través de este estudio de caso pretendemos conocer el tipo de enseñanza que recibe una alumna en el aula de inglés de un instituto público de educación secundaria obligatoria. La alumna a la que en adelante nos referiremos con el nombre ficticio de Atenea de 16 años y nacionalidad española presenta discapacidad intelectual leve. El objetivo general de este estudio es determinar qué aspectos de la enseñanza contribuyen a su aprendizaje y cuáles lo dificultan. Con este propósito, hemos analizado las adaptaciones metodológicas y material didáctico que se utilizan para la enseñanza de Atenea, y hemos estudiado la valoración de las profesoras de inglés de la alumna con respecto a sus condiciones de trabajo en el aula.

\section{Preguntas de investigación}

Nuestro estudio pretende dar respuesta a las siguientes preguntas:

1. ¿Qué adaptaciones y materiales didácticos se emplean para facilitar el aprendizaje de inglés por parte de Atenea? 
2. ¿Qué enfoques metodológicos y dinámicas de clase se emplean para facilitar el aprendizaje de Atenea en el aula de inglés?

3. ¿Cuál es la valoración de las profesoras de inglés de Atenea con respecto a sus condiciones de trabajo en el aula?

\section{Marco teórico}

\subsection{La atención al alumnado con discapacidad intelectual leve: un cambio de paradigma}

La Asociación Americana de Psiquiatría define la discapacidad intelectual como "un trastorno que comienza durante el período de desarrollo y que incluye limitaciones del funcionamiento intelectual como también del comportamiento adaptativo en los dominios conceptual, social y práctico" (American Psychiatric Association, 2013: 17). En esta definición se alude a los déficits cognitivos, pero también a la capacidad de adaptación del individuo según su contexto sociocultural. Como señala Tamarit, esta definición de la discapacidad va enfocada a determinar los apoyos necesarios para la persona "partiendo de su contexto social y familiar, su biografía, sus competencias comunicativas, cognitivas, sociales, la capacidad de regulación de su comportamiento, la presencia de condiciones de salud mental y la presencia o no de condiciones de salud física" (ibídem, 2017: 415), y tiene su raíz en un cambio de paradigma progresivo que se remonta a 1992 y que fue encabezado por la Asociación Americana de Discapacidades Intelectuales y del Desarrollo y las corrientes de la psicología positiva, entre las que es destacable la figura de Martin Seligman. Dicho cambio de paradigma, de enorme repercusión mundial, supone el abandono de la concepción aristotélica de la discapacidad centrada en el déficit o en el "todo para la persona sin la persona", para dejar paso a una nueva conceptualización de la discapacidad en la que la relación entre inteligencia y apoyo no es unívoca, sino que se tienen en cuenta tanto las debilidades como las fortalezas de la persona, se le otorga mayor relevancia al entorno, se profundiza en el progreso de todas las personas con los apoyos adecuados y se sitúa a la persona como eje y garante en la construcción de su destino, lo que viene a ser el "todo para la persona con la persona" (ibíd., 2017). A la luz de la concepción de la discapacidad expuesta, nos centraremos a continuación en los rasgos fundamentales de la discapacidad intelectual leve de acuerdo con la edición publicada en 2013 del Diagnostic and Statistical Manual for Mental Disorders (DSM-5) por la Asociación Americana de Psiquiatría. En este sentido, ha de aclararse que dicho documento establece tres niveles en la clasificación de la discapacidad intelectual: leve, moderada y grave.

En el dominio conceptual, las personas con discapacidad intelectual leve presentan dificultades en el aprendizaje en relación con la lectura, la escritura, la aritmética, el tiempo y el dinero. Asimismo, manifiestan una alteración en la capacidad de pensamiento abstracto, al igual que en la función ejecutiva (planificación, definición de estrategias, determinación de prioridades y flexibilidad cognitiva) y en la memoria a corto plazo.

En el dominio social, se caracterizan por su inmadurez en lo que se refiere a las relaciones sociales. En lo que respecta a la comunicación, conversación y lenguaje suelen ser más restringidos e inmaduros, lo que repercute en su capacidad para valorar y comprender el riesgo en determinadas situaciones en lo que concierne al juicio social. Este hecho conlleva que las personas con discapacidad intelectual leve puedan ser manipuladas. 
En el dominio práctico, pueden precisar ayuda en tareas complejas de la vida cotidiana. El cuidado personal lo realizan de manera adecuada. Los hobbies y áreas de interés suelen ser similares a las de los grupos de su edad, aunque a menudo necesitan ayuda en el juicio respecto al bienestar y la gestión del ocio. En la etapa adulta, estas personas requieren ayuda en actividades como la compra, el transporte, la organización doméstica, el cuidado de los hijos, el cuidado de la salud y la gestión legal y bancaria.

\subsection{Revisión de la literatura en torno a la enseñanza de lenguas para estudiantes con discapacidad intelectual leve}

El estudio de una lengua extranjera resulta una tarea de especial dificultad para todo el alumnado debido mayormente a la complejidad y variedad de procesos mentales que tienen lugar de forma simultánea (Ehrman, 1996). De manera que si aludimos concretamente al alumnado con discapacidad intelectual leve, la dificultad que presenten a la hora de aprender una segunda lengua será mucho mayor -dadas las dificultades referenciadas en el apartado anterior-, sobre todo en lo referente a los procesos cognitivos relacionados con la memoria, atención, motivación y gestión de las emociones. Sin embargo, los numerosos beneficios que conlleva el aprendizaje de una LE desde el punto de vista cognitivo, metacognitivo, lingüístico y sociocultural han sido puestos de manifiesto por diversos estudiosos a lo largo de distintos programas de intervención en los que participaron niños y adolescentes que presentaban distintas dificultades de aprendizaje relacionadas con el lenguaje y cognición (Bruck, 1978; Bacon, 1998; Hurst, 1996; Sparks et al. 1996). Asimismo, algunos autores han demostrado que el alumnado, independientemente del tipo de dificultad que presente, si cuenta con el apoyo pedagógico adecuado, puede alcanzar resultados similares a los de sus compañeros (Kleinert et al, 2007; Tolbert et al., 2015).

De manera general, numerosos estudios existentes abordan las dificultades en los procesos de enseñanza-aprendizaje de lenguas extranjeras en categorías amplias como "foreign language difficulties", "foreign language disabilities" o "Special Educational Needs and foreign language learning". En dichas categorías se engloba a todo el alumnado con dificultades en el aprendizaje de una segunda lengua o una LE, incluyendo alumnado con problemas cognitivos, de lenguaje y comunicación, problemas derivados de discapacidades físicas y problemas sociales. A continuación, presentamos una relación de propuestas de adaptaciones metodológicas que han resultado ser beneficiosas para el alumnado catalogado bajo las categorías mencionadas. Si bien las propuestas no van dirigidas exclusivamente al alumnado con discapacidad intelectual leve, es imprescindible subrayar que dado que este tipo de alumnado comparte muchas de las áreas de dificultad en términos de memoria, atención, metacognición, conciencia fonológica y habilidades lingüísticas con el alumnado que presenta otro tipo de dificultades, también pueden beneficiarse de dichas propuestas (Reynolds et al., 2013). Entre las adaptaciones metodológicas que la literatura existente recoge, hemos considerado relevante destacar las siguientes:

- Enseñanza basada en el enfoque comunicativo (Mabbot, 1994; Hurst, 1996; Downey y Snyder, 2000): el giro metodológico ocurrido en las últimas décadas en lo que respecta a la enseñanza de lenguas ha sido demostrado beneficioso para el aprendizaje por parte del alumnado con dificultades de aprendizaje. Uno de sus 
puntos fuertes es la variedad de tareas y micro-tareas encaminadas al desarrollo por igual de las destrezas lingüísticas y a la realización de las mismas de manera contextualizada, ofreciendo una gran variedad de situaciones y contextos comunicativos. La variedad de tareas conlleva una mejor adaptación a la diversidad de ritmos y estilos de aprendizaje así como a los distintos intereses. Las actividades de reflexión sobre la lengua que habitualmente tenían un mayor protagonismo en las secuencias didácticas siguen presentes, pero aparecen de manera más simplificada y enfocadas a comunicar atendiendo al estatus de los participantes en la situación de comunicación y a las distintas intenciones y finalidades.

- Enseñanza explícita (o directa) y estructurada con énfasis en la estructura del lenguaje -fonética, sintaxis, gramática y semántica- (Demuth y Smith, 1987; Hill et al., 1995): el alumnado con discapacidad intelectual leve requiere a menudo de mayor atención en lo que respecta al aprendizaje de aspectos de la lengua con mayor demanda cognitiva. Por ello, la presentación y secuenciación de los mismos debe estar estructurada de manera coherente y sistematizada, de manera que el alumnado pueda seguir la exposición sin dificultad y la progresión de los contenidos se base en sus conocimientos previos partiendo del contexto cercano hacia un contexto más lejano, avanzando de lo simple a lo concreto, de lo general a lo específico.

- Método Total Physical Response (TPR), (Kleinert et al., 2007; Mohammadian, 2016): el alumnado puede mejorar el almacenamiento de información a través de la asociación que se establece entre movimiento y memoria. Este método contribuye al incremento de la memoria en el alumnado con discapacidad intelectual y facilita un canal que promueve el empleo de técnicas basadas en la afectividad.

- Enseñanza multi-sensorial (Sparks y Miller, 1998; Amend et al., 2009; Tolbert et al., 2015): la combinación de canales auditivos, visuales y kinestésicos facilita el aprendizaje de la LE. Cuanto más elaborada y sustentada esté la información de entrada a través de los distintos canales, más sencillo será el retener dicha información. Este tipo de enseñanza favorece la memoria y atención del alumnado con discapacidad intelectual leve y favorece la creación de un clima de clase más distendido con respecto al tradicional.

- Enseñanza basada en el enfoque de las inteligencias múltiples (Gardner, 1993; Fonseca, 2004): el empleo de secuencias didácticas que combinen el uso de diversos tipos de inteligencia facilita la adaptabilidad del material curricular a los distintos estilos de aprendizaje. Esto repercute positivamente en la atención y motivación del alumnado con discapacidad intelectual leve.

- Explicitación de estrategias de aprendizaje (McColl, 2000; Difino y Lombardino (2004); Kleinert et al., 2007): aprender a aprender resulta imprescindible para el alumnado con discapacidad intelectual leve sobre todo en aquellos casos en que o bien se encuentra sobreprotegido o bien no está lo suficientemente atendido. Explicitar el uso de estrategias de aprendizaje contribuye a que este alumnado adquiera poco a poco una cierta autonomía en su aprendizaje. Entre dichas estrategias, los autores anteriormente indicados señalan: repetición coral, uso de patrones de color para el aprendizaje de aspectos lingüísticos, revisión y repetición frecuentes, uso de guías y organizadores...

- Aspectos afectivos y clima de la clase (Dörnyei y Skehan, 2003; Mohammadian, 2016): la puesta en práctica encaminada al desarrollo y comprensión de aspectos 
afectivos vinculados a las emociones y los sentimientos, promueven la creación de un clima positivo de aprendizaje para todo el alumnado, incluyendo al alumnado con discapacidad intelectual leve que, a menudo, debe lidiar con problemas de ansiedad, autoestima, personalidad, inhibición y motivación como consecuencia de su discapacidad.

- Docencia compartida (Comisión Europea, 2005; Scruggs et al., 2007): el hecho de que haya dos profesores en una misma clase favorece la comunicación y la atención al alumnado con mayores dificultades, puesto que se facilita el seguimiento y retroalimentación los cuales tienen lugar con mayor frecuencia.

- Aprendizaje cooperativo (Sapon-Shevin, 1999; Comisión Europea, 2005): este tipo de aprendizaje favorece la inclusión y motivación del alumnado con discapacidad intelectual leve a través del trabajo y retroalimentación entre iguales, lo que implementado de manera adecuada contribuye tanto a su aprendizaje como autoestima.

- Uso de las Tecnologías de la Información y de la Comunicación (McColl, 2000; Meiring y Norman, 2005; Tolbert et al., 2015): las TIC ofrecen, además de nuevos canales de interacción y mejoras en el seguimiento y feedback, mayores posibilidades de adaptar el material didáctico según niveles de dificultad.

- Evaluación frecuente y uso de distintos tipo de (auto)evaluación (Difino y Lombardino, 2004): los distintos tipos de evaluación existentes manifiestan la flexibilidad y diversidad de herramientas disponibles para la evaluación de los distintos ritmos y estilos de aprendizaje. La autoevaluación, por su parte, es imprescindible en la adquisición de la competencia clave de aprender a aprender del alumnado y, más aún, en aquel con discapacidad intelectual que a menudo presenta dificultades en cuanto a autodeterminación, principalmente en lo que concierne a discernir cuáles son sus fortalezas y debilidades en el proceso de aprendizaje.

De manera más específica, en el ámbito internacional, hemos observado estudios muy recientes que consisten en programas de intervención a través de una determinada metodología, técnica o estrategia con el alumnado con discapacidad intelectual leve. Uno de ellos pone de manifiesto los efectos beneficiosos del aprendizaje del inglés como lengua extranjera a través del trabajo de la afectividad con el alumnado con discapacidad intelectual y basándose en el método Total Physical Response (Mohammadian, 2016). Otro de los estudios se centra en la implementación de una herramienta tecnológica denominada Talking Tactile Technology (T3) que se caracteriza por combinar varios canales: visual, auditivo y kinestésico, en alumnado principiante con discapacidad intelectual leve de inglés como primera lengua extranjera arrojando resultados positivos (Coskun, 2013). En esta dirección apunta otro estudio que contrapone el uso del canal audio-visual al uso único de medios visuales para la enseñanza del vocabulario (Noori y Farvardin, 2015). Asimismo, resulta de especial relevancia aludir a una investigación centrada en el análisis de material didáctico, más concretamente, de libros de texto de inglés para alumnado adolescente en un centro de educación especial de Irán. El análisis del material se basaba en observaciones de clase complementado con la elaboración de una escala Likert de 5 puntos destinada a ser cumplimentada por el investigador. Dicho estudio sintetiza sus ventajas e inconvenientes y concluye que los libros de texto utilizados no son adecuados para el aprendizaje del inglés en este tipo de alumnado 
(Ghanaat y Fahimniya, 2015). ${ }^{1}$ Por último, otro estudio puso de relieve que las diferencias individuales y la acción pedagógica jugaban un papel determinante en el aprendizaje de la LE del alumnado con discapacidad intelectual leve (Krapez, 2012). Pese a la importancia del tema que nos concierne, en nuestro país no hemos identificado ningún estudio acerca de la enseñanza de inglés como lengua extranjera al alumnado con discapacidad intelectual leve en ninguna etapa de escolarización, lo cual justifica la necesidad de un número mayor de estudios que analicen los procesos de enseñanza-aprendizaje del inglés lengua extranjera en las distintas etapas de escolarización y contextos en este tipo de alumnado.

\section{Metodología}

\subsection{Descripción del centro}

El centro escolar donde se ha llevado a cabo el estudio de caso es un Instituto de Educación Secundaria Obligatoria de titularidad pública situado en una población de aproximadamente 20.000 habitantes del área metropolitana de Granada. El centro se inauguró en el curso académico 2003-2004. Inicialmente se caracterizó por llevar a cabo un proyecto de innovación centrado en la atención al alumnado con síndrome de Down que fue aprobado por la Consejería de Educación de la Junta de Andalucía. Desde entonces, su principal seña de identidad en el contexto provincial y regional ha sido su modelo de atención a la diversidad, realizado bajo un enfoque inclusivo. La puesta en práctica de dicho enfoque se ha visto obstaculizada en algunas etapas de su andadura, entre otras razones por la fluctuación del profesorado. Actualmente, la plantilla estable no llega al $60 \%$, por lo que la plantilla de profesorado que fluctúa constantemente representa más de un $40 \%$.

El centro cuenta con 550 estudiantes, de entre los cuales 44 de ellos -es decir, el 8\%-, presentan necesidades específicas de apoyo educativo. A su vez, según declaraciones del director del centro, existe un porcentaje de estudiantes que aún no han sido diagnosticados $y$, sin embargo, presentan deficiencias en su aprendizaje.

\subsection{Participantes}

El estudio de caso realizado se centra en torno a una alumna con discapacidad intelectual leve, incluyendo su entorno escolar y familiar. De este modo, los participantes en el estudio son:

- Atenea: alumna con discapacidad intelectual leve.

- Compañeros de Atenea en la clase de inglés.

- Profesoras de inglés de Atenea (docencia compartida durante los cursos de $3^{\circ}$ y $4^{\circ}$ ).

- Equipo directivo.

- Familiares de Atenea.

\footnotetext{
${ }^{1}$ Si se desea ampliar esta información consúltese: Ghanaat, S., y Fahimniya, F. (2015). "An evaluation of English language textbooks of mild/educable mentally retarded secondary school students in Iran", en Indian Journal of Fundamental and Applied Life Sciences, 5: $830-843$.
} 


\subsection{Planteamiento y desarrollo del estudio e instrumentos de recogida de datos}

Hemos realizado un estudio longitudinal durante dos cursos académicos en los cuales Atenea ha cursado respectivamente $3^{\circ}$ y $4^{\circ}$ de ESO. Como corresponde a la praxis propia del estudio de caso (Stake, 1998), hemos realizado un estudio en profundidad de la persona y su entorno, con el fin de adentrarnos en la complejidad de las necesidades de aprendizaje de la estudiante objeto de nuestro estudio, así como en las respuestas educativas ofrecidas. Hemos de subrayar la importancia de este tipo de estudio para arrojar luz sobre un fenómeno tan escasamente estudiado como lo es el que abordamos en la presente investigación. Si bien no podemos afirmar que debido a la naturaleza propiamente etnográfica del estudio los resultados de nuestro trabajo sean generalizables, sin duda, como sostiene Merriam (2009), este tipo de estudios arrojan luz sobre una realidad educativa que puede darse en alumnado de las mismas características en contextos similares.

Para asegurar la validez y confiabilidad del estudio de caso, hemos elaborado los instrumentos de recogida de datos basándonos en el criterio de expertos que han analizado nuestras propuestas $\mathrm{y}$, a su vez, basándonos en nuestro propio criterio atendiendo a las necesidades derivadas de la investigación. Posteriormente, hemos triangulado los datos obtenidos a través de los distintos instrumentos empleados: observación participante, entrevistas en profundidad, reuniones formales e informales y análisis documental.

La observación participante ha sido realizada por parte de los investigadores en calidad de voluntarios en el aula de inglés alternándose su asistencia por trimestres. Durante los dos años del estudio se han elaborado sendos cuadernos del investigador donde se han recogido las notas de la observación registradas a mano que posteriormente han sido puestas en discusión en reuniones trimestrales con el fin de facilitar su análisis. El foco de atención han sido las actuaciones de Atenea, la metodología seguida por parte del profesorado, la actuación del resto de alumnos y alumnas de clase, y otros datos incidentales. Paralelamente, y con carácter semanal, se acordó impartir clases de apoyo a la alumna de una hora de duración en horario extraescolar en el domicilio de la alumna por uno de los investigadores. El objetivo de dicha intervención, además de reforzar el aprendizaje de Atenea con el fin de lograr su éxito académico en la asignatura, iba orientado a profundizar en sus procesos cognitivos y afectivos, así como a la comparación de los datos obtenidos en clase.

Para la elaboración de las entrevistas nos hemos basado en el Modelo Socio-Cultural de Gardner y MacIntyre (1993), que ya fue adaptado por Viskari (2005) como marco teórico para analizar los diversos factores que operan específicamente en el proceso de aprendizaje de un idioma extranjero por parte del alumnado con dificultades de aprendizaje (véase Viskari 2005, pp. 12-46). Se trata de entrevistas en profundidad, las cuales incluyen tanto preguntas cerradas como de libre respuesta. Las entrevistas fueron grabadas en formato de audio y posteriormente transcritas. Basándonos en las guías metodológicas de Miles, Huberman y Saldaña (2014), los datos obtenidos han sido analizados por los dos investigadores a partir de tres lecturas profundas de las transcripciones en intervalos de diez días, lo que nos ha permitido codificar categorías temáticas con un grado de objetividad alto teniendo en cuenta todos los matices posibles gracias al contraste de los puntos de vista de los investigadores. Este análisis comparativo ha permitido desmenuzar los datos extraídos en partes para su posterior codificación en temas emergentes.

Asimismo, durante los dos cursos académicos ambos investigadores hemos mantenido

\section{4}


reuniones formales e informales con el equipo docente y el equipo directivo, así como con el profesorado y la familia de Atenea que han contribuido a la toma de decisiones y a la interpretación de los datos recabados. Por último, el análisis documental ha sido una herramienta esencial en nuestra investigación. Se incluyen aquí el estudio del material didáctico en su implementación, los documentos propios del centro (Proyecto Educativo de Centro y Reglamento de Organización Interna), la historia de salud de Atenea, su expediente académico, el dictamen de escolarización y el informe psicopedagógico.

\section{Análisis de datos y Resultados}

1. ¿Qué adaptaciones y materiales didácticos se emplean para facilitar el aprendizaje de inglés por parte de Atenea? Fortalezas y debilidades analizadas.

En lo que concierne a las adaptaciones y materiales didácticos empleados con Atenea, la alumna dispone de un dossier de actividades extraídas y adaptadas por cada una de las unidades didácticas procedentes del libro de texto empleado en clase. Los libros de texto de los que se desprende el material adaptado son Build up 3 para tercero de ESO y Build up 4 para cuarto de la editorial Burlington. El enfoque comunicativo es el que predomina mayormente a lo largo de las 9 unidades didácticas que conforman el manual y que se caracteriza por la inclusión de actividades destinadas al desarrollo de las 4 destrezas lingüísticas. El material adaptado procede de digitalizaciones del libro de texto y de su posterior modificación conjunta por parte del departamento de inglés del centro utilizando para ello como herramienta un procesador de textos. Dichas digitalizaciones conforman un dossier por cada una de las unidades didácticas. En algunas tareas, el dossier remite a la alumna al libro de texto para que las realice utilizando algún recurso textual o visual que no esté contenido. Además del dossier por unidad Atenea dispone, al igual que sus compañeros, del programa de lectura adaptado por niveles Raz-kids. El programa se emplea como recurso complementario para el trabajo de la compresión escrita y debe ser utilizado como tarea para la casa. Por último, hemos observado que el material adaptado del que dispone Atenea es el mismo que se emplea para el alumnado con necesidades educativas especiales derivadas de síndrome de Down, el resto del alumnado trabaja con el libro de texto y un cuaderno de clase.

Con el objetivo de facilitar la comprensión de este apartado, tras el análisis y puesta en común de los resultados obtenidos por parte de los dos investigadores, hemos considerado pertinente el responder a las preguntas de investigación a través de las fortalezas y debilidades que presentan los materiales analizados. Para ello, nos hemos basado en una evaluación de elaboración propia basada en criterios puestos en diálogo permanente con aquellos de expertos y los recogidos tras la revisión de la literatura.

Las fortalezas del material adaptado son las siguientes:

- Reducción de la carga cognitiva a través de la selección y adaptación de las actividades del libro más representativas.

- Explicitación de la gramática y el vocabulario que debe adquirirse durante el desarrollo de cada unidad didáctica a partir de tablas, gráficos y actividades de asociación de imágenes con determinadas unidades léxicas. 
- Inclusión de actividades destinadas al desarrollo de las cuatro destrezas lingüísticas, así como de gramática, vocabulario y reflexión sobre la propia lengua.

- Descripción sintética de los contenidos que se van a trabajar al inicio de cada unidad didáctica.

- Fomento del gusto por la lectura, la comprensión y expresión escrita y el aprendizaje de contenidos complementarios al currículum a través del uso del programa Raz-kids. Dicho programa emplea un método de lectura multinivel y vehicula un canal de interacción audiovisual que facilita al profesorado ILE la asignación, el seguimiento y la evaluación de lecturas de manera individual.

A continuación exponemos las debilidades del material adaptado de Atenea:

- Visibilidad y legibilidad escasas. En determinadas actividades, la fuente es demasiado pequeña. A su vez, muchas de las imágenes que contiene el dossier no se aprecian adecuadamente debido al tamaño o al hecho de que están representadas en blanco y negro.

- Primacía de actividades de gramática y vocabulario en detrimento de actividades más comunicativas y contextualizadas. Primacía de actividades de traducción del inglés al español (en torno a un $35 \%$ de las actividades). Varias actividades presentadas como actividad de comprensión escrita están destinadas al ejercicio de la traducción.

- Las instrucciones, guías y enunciados no se presentan de manera precisa. Esto impide el seguimiento y la comprensión del desarrollo de la unidad didáctica así como su finalidad por parte de cualquier lector. Asimismo, la mayor parte de instrucciones, guías y enunciados están redactados en español. Normalmente dichos enunciados no se incluyen en inglés. Cuando se incluyen, hemos detectado que no siempre el enunciado en español se corresponde con el enunciado de la misma actividad en inglés. Además, no suele haber enumeración ni delimitación clara de los apartados de las unidades didácticas, y el orden de las actividades y su enumeración a veces son erróneos o confusos.

- En una gran parte de las actividades, el uso de los patrones de color tanto para la enseñanza de la gramática y vocabulario como para la exposición de instrucciones no es lo suficientemente preciso ni coherente; se emplea al azar y de manera desordenada.

- No hemos observado ninguna actividad destinada a la autoevaluación. Ej.: uso del portfolio.

- No hemos apreciado que en el dossier se explicite el uso de estrategias de aprendizaje ni se propongan ni se faciliten canales audiovisuales y/o kinestésicos para afianzar mejor el aprendizaje.

2. ¿Qué enfoques metodológicos y dinámicas de clase se emplean para facilitar el aprendizaje de Atenea en el aula de inglés? Fortalezas y debilidades analizadas.

La metodología observada es ecléctica. Se combinan elementos propios del enfoque comunicativo con otros propios de una metodología tradicional. Estos últimos tienen mayor peso en la dinámica del aula, especialmente en lo que respecta al alumnado con NEE. En 
este sentido, la clase se dedica principalmente a la explicación de la gramática y vocabulario y a la realización de las actividades del libro de texto. No obstante, con carácter semanal o quincenal se introducen actividades más dinámicas y cooperativas para el trabajo de la expresión y comprensión oral, por ejemplo: representación de diálogos; juegos; canciones... Al ser una clase donde la docencia es compartida, una de las profesoras por lo general adopta un rol instructivo y la otra adopta un rol de apoyo a las dificultades que surjan.

Entre las fortalezas detectadas se incluyen los siguientes aspectos:

- Realización de tareas de aprendizaje cooperativo de manera semanal o quincenal.

- Programación y evaluación multinivel general.

- Docencia compartida por dos profesoras dentro de clase, inclusión de personal como becarios y voluntarios.

- Uso del programa Raz-kids.

- En la evaluación continua se valora el desarrollo de las cuatro destrezas de manera individual y grupal.

- Con respecto a la organización del alumnado, este está dispuesto en el espacio de clase en grupos de trabajo (working groups).

Las debilidades detectadas son las siguientes:

- No hay distinción en la forma de aplicar la metodología adaptada para el alumnado con NEE incluso cuando dentro de esta categoría se presentan perfiles de aprendizaje distintos.

- Mayor peso de la metodología tradicional para el alumnado con NEE.

- La prueba escrita dirigida al alumnado con NEE no incluye la evaluación de las cuatro destrezas.

- El alumnado con necesidades en general, y Atenea en particular, se encuentra a menudo desconectado del funcionamiento del grupo clase, y recibe muy poco feedback durante las correcciones. Esto es así porque, a diferencia de sus compañeros, Atenea no cuenta con el working plan o guía de trabajo en la que se pautan las actividades y la fecha límite para su realización.

- Se etiqueta al alumnado con necesidades. Al referirse a ellos se usan términos como "los adaptados" o "los que tienen necesidades".

- Funcionamiento de los grupos de trabajo poco adecuado. Ausencia de espíritu de equipo. Se trabaja gran parte del tiempo de manera individual. Los grupos están establecidos de manera que en cada uno de ellos haya alumnado de distintas capacidades, con objeto de que los estudiantes con necesidades sean ayudados por sus compañeros y compañeras. Esto hace que la responsabilidad del aprendizaje de los estudiantes con dificultades en general, y el de Atenea en particular, recaiga sobre el resto, quienes a menudo se limitan a darles la respuesta correcta. Así lo confirma una de las profesoras en su entrevista:

"Pues con ella puede haber varios momentos de atención y también de olvidarla por completo [...] hay veces en las que sí la atienden, sí la ayudan, sí la integran $\mathrm{y}$, sobre todo, hay algunos alumnos que si te ven que tú estás mirando a lo mejor 
le prestan más atención porque consideran que tienen que... pero hay momentos en los que se olvidan totalmente y la dejan allí y ellos están hablando entre ellos y la dejan allí aparcada porque ella tampoco hace por...pero que son ellos los que de alguna manera no pueden dejarla a un lado y están con sus conversaciones a veces de dos en dos o de tres en tres y ella pues está...olvidada".

Esta declaración evidencia la gran responsabilidad que se impone al resto del alumnado de clase, pues no hablamos de integración solamente, sino de asumir una función fundamental en su aprendizaje.

Tras haber analizado los dossiers de Atenea, hemos observado que la mayoría de las actividades están sin corregir y muchas de ellas también sin completar, bien porque la alumna no ha asistido a clase o bien porque no se le han revisado ni se le ha pedido que las realice. Por tanto, podemos afirmar que Atenea no ha obtenido un feedback ni seguimiento rutinario a lo largo de los dos cursos más que el que proviene de la clase de refuerzo impartidas por los voluntarios (investigadores de este trabajo) o de los momentos en que algún voluntario o profesora le ayuda con alguna actividad.

3. ¿Cuál es la valoración de las profesoras de inglés de Atenea con respecto a sus condiciones de trabajo en el aula?

Las profesoras resaltan como puntos fuertes su "entusiasmo por la enseñanza, el acercamiento al alumnado y la creatividad". Asimismo, mencionan como una acción importante la elaboración propia del material didáctico adaptado a partir del libro de texto, que realiza anualmente el departamento de inglés, de forma que el alumnado con necesidades "tenga un material adecuado y asequible a sus posibilidades de aprendizaje", tarea que "no es fácil, pues requiere su tiempo ver cómo aprenden y adaptar las actividades a la forma en la que ellos aprenden".

No obstante, señalan una serie de dificultades encontradas, las cuales son una fuente de ansiedad y estrés, pues sienten que los recursos que tienen son claramente insuficientes para dar respuesta a las necesidades individuales de su alumnado. Estas dificultades son:

- Ratio de alumnado muy elevada, lo cual les impide implementar una metodología más activa, y las lleva a recurrir a menudo a la traducción.

- Carencia de herramienta digital que facilita la enseñanza personalizada: "en una clase diversa es fundamental tener disponibles tabletas con sus auriculares para trabajar"; "a mí me gustaría que los alumnos dispusieran de una Tablet, por ejemplo, porque sería un avance extraordinario, podría personalizar las lecturas... ¡todo! Podría programar con ellos todas las actividades y la enseñanza sería mucho más eficaz".

- Necesidad de más personal de apoyo, sobre todo en lo que respecta a la creación del material didáctico.

- Falta de información sobre los perfiles individuales del alumnado con dificultades de aprendizaje.

Las profesoras ponen de manifiesto la dificultad que conlleva ofrecer una atención 
equitativa y de calidad a todo el alumnado. La incapacidad de atender adecuadamente al alumnado con necesidades educativas especiales les causa frustración y malestar: "es un esfuerzo y sobre todo una frustración porque no les llego a todos [...] me produce estrés y ansiedad muchísima porque pienso que, si no, estos alumnos están ahí sentados mirando y no reciben mi ayuda". Inciden en el hecho de que las dificultades se incrementan al tratarse de una clase de idioma extranjero: "es una materia que la tienes que practicar muchísimo, que no es teórica, que es continuamente utilizar el idioma...”.

En relación con todo lo anterior, la enseñanza de refuerzo impartida a Atenea por parte de los investigadores ha puesto de manifiesto que el avance de la alumna en el aprendizaje del inglés es notable cuando:

- Atenea comprende claramente los objetivos de aprendizaje y las tareas que ha de desarrollar;

- realiza las actividades y trabaja de manera continuada;

- se corrigen sus actividades y se solventan sus dudas cuando lo necesita;

- se despierta su motivación e interés, fundamentalmente a través de la percepción de su propio progreso;

- se la hace responsable de su propio aprendizaje y se siente valorada.

El análisis de la entrevista mantenida con Atenea pone de relieve la importancia de sentirse apreciada por sus profesores y profesoras. Según Atenea, el hecho de sentir a una profesora "cercana", "que explica bien" y "que es simpática" tiene una importante incidencia en su motivación e interés.

Finalmente, se evidencia el papel fundamental de la familia. Como declara una de las profesoras, la asistencia de Atenea a clase no es regular, lo cual tiene un papel decisivo en su aprendizaje. La propia madre de Atenea reconoce haberla sobreprotegido. Si bien parte de estas ausencias están justificadas en función de la salud de Atenea, en numerosas ocasiones obedecen más al hecho de que, en palabras de la madre de Atenea, "la familia la ha sobreprotegido", y no obedecen a una causa de peso.

\section{Conclusiones}

De manera global, tras el estudio de las adaptaciones y enfoques metodológicos podemos afirmar que se necesitan una serie de mejoras con el fin de que su implementación sea adecuada. En este sentido, los datos obtenidos apuntan hacia la necesidad de una mejora en la dotación y gestión de recursos humanos e infraestructurales, una profundización en el ámbito pedagógico en lo que respecta a la formación del profesorado, colaboración departamental, interdepartamental e incluso entre distintos centros, colaboración con la comunidad, con centros universitarios y de investigación, e implementación de políticas educativas que hagan posible la consecución de los objetivos propuestos en materia de inclusión. Todo ello resulta imprescindible para responder de manera efectiva a la diversidad de necesidades, estilos y ritmos de aprendizaje presentes en el aula de LE. Nuestro estudio ha puesto de relieve las carencias existentes al respecto, las cuales han de ser suplidas por la buena voluntad del profesorado. 
Se hacen necesarios estudios sobre la enseñanza de lenguas extranjeras a alumnado con discapacidad intelectual, en particular trabajos que realicen un análisis profundo de situaciones y contextos, de forma que puedan salir a la luz las necesidades reales en materia de formación y dotación de recursos.

\section{REFERENCIAS}

Amend, A.E., Whitney, C.A., Messuri, A.T., y Furukawa, H. (2009). “A modified sequence for students with language-based learning disabilities". Foreign Language Annals, 42, 1: 27-41.

American Psychiatric Association (2013). Diagnostic and Statistical Manual of Mental Disorders, Fifth Edition. Arlington, VA: American Psychiatric Association.

Bacon, S. (1998). "Conceptual knowledge, biliteracy, and the at-risk child", en M. Met (ed.), Critical issues in early second language learning. Glenview, IL: Scott Foresman-Addison Wesley, 313-318.

Bruck, M. (1978). "The suitability of early French immersion programs for the language disabled child" en Canadian Journal of Education, 3: 51-72.

Coskun, A. (2013). "Teaching English to non-native learners of English with mild cognitive impairment", en Modern Journal of Language Teaching Methods, 3, 4: 8-16.

Declaración Universal de Derechos Humanos (1948): Resolución 217 A (III) de la Organización de las Naciones Unidas. París (Francia), 10 de diciembre de 1948.

Demuth, K.A., y Smith, F. (1987). "The foreign language requirement: An alternative program", en Foreign Language Annals, 20, 1: 67-77.

Difino, S. y Lombardino, L. (2004). "Language learning disabilities: The ultimate foreign language challenge", en Foreign Language Annals, 37, 3: 390-400.

Dörnyei, Z., y Skehan, P. (2003). "Individual differences in second language learning", en C.J. Doughty, y M.H. Long (eds.), The handbook of second language acquisition. Oxford: Blackwell, 589-630.

Downey, D.M., y Snyder, L.E. (2000). "College students with LLD: The phonological core as risk for failure in foreign language classes", en Topics in Language Disorders, 21: 82-92.

Ehrman, M.E. (1996). Understanding second language learning difficulties. Thousand Oaks, CA: SAGE Publications, Inc.

European Commission (2005). Insights and Innovation. Special Educational Needs in Europe. The teaching and Learning of Languages. Finland: European Commission.

Fonseca Mora, M.C. (2004). "Multiple Intelligences Theory as a framework for teaching English to teenagers in Spanish context", en P. Montijano A TEFL Methodology. Málaga: Aljibe

Gardner, H.E. (1993). Multiple intelligences: The theory in practice. New York: Basic Books.

Gardner, R.C., y MacIntyre, P.D. (1993). “A student's contributions to second language learning. Part II: Affective variables" en Language Teaching 26, 1-11.

Ghanaat, S., y Fahimniya, F. (2015). "An evaluation of English language textbooks of mild/educable mentally retarded secondary school students in Iran", en Indian Journal of Fundamental and Applied Life Sciences, 5: 830-843.

Hill, B., Downey, D., Sheppard, M., y Williamson, V. (1995). Accomodating the needs of students with severe language learning difficulties in modified language classes. Broadening the frontiers for foreign language instruction. Lincolnwood, IL: National Textbook.

Hurst, D. (1996). "Teaching Spanish to children with different learning styles: evolution of philosophy", en Hispania, 79, 1: 123-125. 
Kleinert, H.L., Cloyd, E., Rego, M., y Gibson, J. (2007). "Students with disabilities: Yes, foreign language is important", en Teaching Exceptional Children, 39, 3: 24-29.

Krapez, S. (2012). "Second Language Comprehension and Acquisition in Mentally Disabled Children- Illusion or Reality”, en IBS Newsletter porocevalec. Disponible en http://porocevalec. ibs.si/en/component/content/article/42-letnik-1-t-3/136-staa-krape-ma-ed-applied-linguisticssecond-language-comprehension-and-acquisition-in-mentally-disabled-children-illusion-orreality. [consultado: 28 de junio, 2017].

Mabbot, A. (1994). "An exploration of reading comprehension, oral reading errors, and written errors by subjects labeled learning disabled", en Foreign Language Annals, 27, 3: 293-324.

McColl, H. (2000). Modern languages for all. London: David Fulton Publishers.

Ley Orgánica 2/2006, de 3 de mayo, de Educación. Jefatura del Estado. "BOE" núm. 106, de 4 de mayo de 2006.

Ley Orgánica 8/2013, de 9 de diciembre, para la Mejora de la Calidad Educativa. Jefatura del Estado. "BOE" núm. 295, de 10 de diciembre de 2013.

Meiring, L., y Norman, N. (2005). "How can ICT contribute to the learning of foreing languages by pupils with SEN?", en Support for Learning, 20, 3:129.134.

Merriam, S. B. (2009). Qualitative research: A guide to design and implementation. San Francisco, CA: Jossey-Bass.

Miles, M. B., Huberman, A. M., y Saldaña, J. (2014). "Fundamentals of qualitative data analysis", en M. B., Huberman, A. M., y Saldaña, J. (eds.). Qualitative data analysis: A methods sourcebook. Los Angeles, CA: Sage, 69-104.

Mohammadian, A. (2016). "The effect of affection on English language learning of children with intellectual disability based on a total physical response method of language teaching", en International Journal of English Language and Literature Studies, 5, 2: 92-103.

Moore, F.X. (1995). Section 504 and the Americans with Disabilities Act: "Accomodating the learning disabled student in the foreign language curriculum”, en ADFL Bulletin, 26.

Noori, Z.S., y Farvardin, M.T. (2016). "The effect of using audio-visual aids versus pictures on foreign language vocabulary learning of individuals with mild intellectual disability", en Journal of Special Education and Rehabilitation, 17, 1-2: 16-35.

Reynolds, T., Zupanick, C.E., y Dombeck, M. (2013). "Effective teaching methods for people with intellectual disabilities". Disponible en https://www.mentalhelp.net/articles/effectiveteaching-methods-for-people-with-intellectual-disabilities/. Última consulta: 27 de junio, 2017.

Sapon-Shevin, M. (1999). Because we can change the world: A practical guide to building cooperative, inclusive classroom communities. Boston: Allyn and Bacon.

Scruggs, T.E., Mastropieri, M.A., McDuffie, K.A. (2007). "Co-teaching in inclusive classrooms: A metasynthesis of qualitative research", en Council for Exceptional Children, 73, 4: 392-416.

Skinner,M., y Smith, A. (2007). "Designing foreing language instruction for students with learning disabilities: "Modify" or "Substitute"?", en $29^{\text {th }}$ International Conference on Learning Disabilities, Council for Learning Disabilities, Myrtle Beach, SC.

Sparks, R.L., Artzer, M., Patton, J., Ganschow, L., Miller, K., Hordubay, D.J. y Walsh, G. (1998). "Benefits of multisensory language instruction for at-risk foreign language learners: A comparison study of high school Spanish students" en Annals of Dyslexia, 48: 239-270.

Stake, R. E. (1998). Investigación con estudios de caso. Madrid: Morata.

Tamarit. J. (2017). "Las personas con discapacidad intelectual". En A. Marchesi, C.Coll y J. Palacios (eds.), Desarrollo psicológico y educación. Respuestas educativas a las dificultades de aprendizaje y del desarrollo. Madrid: Alianza editorial, 415-443. 
Tolbert, J., Killu, K. y Davis, B. (2015). “A framework for supporting Students with learning disabilities in Spanish courses: Connecting Learning Characteristics and Instructional Methods" en Theory and Practice in Language Studies, 5, 2: 225-234.

Viskari, K. (2005). Foreign language learning disabilities. Theoretical and Practical Tools for English Teachers in Finnish Upper Secondary School. Tesis doctoral. University of Jyväskylä. 